\title{
Inheritance of Powdery Mildew Resistance and Some Economic Traits in Cucumber (Cucumis sativas L.)
}

\author{
Enas S. Khatab, ${ }^{1}$ Sara E. Gomaa, ${ }^{1}$ Abd El-Aziz M. Khalf-Allah, ${ }^{2}$ Aly I. Abido, ${ }^{3}$ Ahmad M. El-Gamal, ${ }^{3}$ \\ Sawsan M. Al-Abed ${ }^{4}$
}

\begin{abstract}
The present study was carried out during successive growing seasons for the years 2014 and 2015, under greenhouse conditions, at Sabahia Horticulture Research Station, Alexandria Governorate, Egypt. Six inbred lines of cucumber were utilized in a half-diallel cross breeding program to obtain $15 F_{1}$ hybrids. The breeding program was concerned with powdery mildew resistance, economic characters and their attributes; i.e. total fruit yield/ plant, number of fruits/plant and average fruit weight; in addition to the fruit quality characteristics; i.e., fruit length and fruit diameter. Results showed that the hybrid $P_{2} \times P_{4}$ gave a very high resistance $(100 \%)$, which is considered an immune (I) hybrid, while, the genotypes $\mathbf{P}_{2}$, $\mathbf{P}_{1} \times \mathbf{P}_{2}, \mathbf{P}_{1} \times \mathbf{P}_{3}, \mathbf{P}_{2} \times \mathbf{P}_{5}$ gave high powdery mildew resistance (HR); i.e., (98.33\%, 98.23\%, 96.3\% and 95.83\%), respectively. The additive gene action exhibited highly significant, positive or negative, values in all the tested crosses for total yield/plant; whereas, the dominance effect values were found to be positively high significant in most crosses. The additive gene action exhibited positive significant values for powdery mildew resistance in most crosses; except the cross $P_{1} \times P_{2}$, which gave a negative high significant values and the two crosses $P_{1} \times P_{5}$ and $P_{2} \times P_{3}$, which gave insignificant values. The dominance gene action was found to have positive high significant values in most crosses for this character.
\end{abstract}

Key words: Cucumber, Powdery mildew resistance, Types of gene action.

Corresponding author: enas15_4@yahoo.com.

\section{INTRODUCTION}

Cucumber (Cucumis sativus L.), $2 \mathrm{n}=(2 \mathrm{x})=14$, belongs to the family Cucurbitaceae, which is an important summer vegetable crop grown in Egypt. Cucumber is originally from Southern Asia (India); nowadays, it is grown worldwide. Many diseases were found to have severe effect on production causing a huge yield loss and declining in the quality. Powdery mildew is considered the most common and serious disease of cucurbit crops in Egypt, it causes serious production problems in both field and greenhouse which, consequently, reduces yield. In Egypt, cucumber powdery mildew intensity decreases highly in autumn than in spring (Abd El-Sayed, 2002). Nevertheless, the early planting results in the lowest percentage of disease intensity. So, plant breeding programs are urgent to produce new hybrids having resistance for this disease to eliminate the damage caused by the infection.

Production of new cucumber hybrids with high quality characters relays on the study and focus on some important traits, especially resistance to important diseases and total yield. Successful hybridization programs depend upon the knowledge regarding the nature of gene action controlling the characters under study to identify both the promising parents and crosses, (Abou Kamer, 2011).

El-Mgihawry et al. (2008) reported that the dominance components ( $\mathrm{H} 1$ and $\mathrm{H} 2$ ) were found highly significant for all cucumber studied traits, and larger in magnitude than the additive effects of the genes (D) indicating the importance of non-additive and additive gene action in the inheritance of days to anthesis of female flower, fruits weight and number of fruits/plant.

The knowledge of the genetic parameters which influences the expression of different quantitative characters is needed to understand the genetic basis of yield and yield components with the information about different gene action which can help the breeder to plan a suitable breeding program to realize the objectives for quality improvement of crop characters. Therefore, the present investigation was carried out to evaluate the degree of resistance of some cucumber genotypes against powdery mildew disease via estimating the types of gene action of $15 \mathrm{~F}_{1}$ - hybrid, resulting from a half-diallel crossing among six inbred lines, to develop new hybrids under greenhouse conditions and to select the top performing ones which are powdery mildew resistant with superior yield and fruit quality.

\section{MATERIALS AND METHODS}

Seeds of 6 different inbred lines of cucumber (Cucumis sativas L.) were kindly provided by the Improvement of the Main Vegetables and Hybrids Production Project, Vegetables branch, Horticulture Research Institute (HRI). The six lines were 9-5-21-23$2 \operatorname{KAHA}\left(\mathrm{P}_{1}\right), 1-26-27-19 \operatorname{KAHA}\left(\mathrm{P}_{2}\right), 67-7-23-128-18$

\footnotetext{
Veg., Medicinal and Aromatic Plants Breeding Dept, Horticulture Research Institute (HRI)

${ }^{2}$ Veg. Dept.,Faculty of Agric., El-Shatby, Alex. Univ.

${ }^{3}$ Plant Production Dept, Faculty of Agric., Saba Basha, Alex. Univ.

${ }^{4}$ Agriculture Botany Dept., Faculty of Agric., Saba Basha, Alex. Univ.

Received April 4, 2016, Accepted April 24, 2016
} 
$\operatorname{DOKKY}\left(\mathrm{P}_{3}\right), \quad$ 1-19-299-2 $\operatorname{KAHA}\left(\mathrm{P}_{4}\right), \quad 25-2-22-15$ $\operatorname{KAHA}\left(\mathrm{P}_{5}\right)$ and 6-5-23-2 $\mathrm{KAHA}\left(\mathrm{P}_{6}\right)$.

Seeds of the six cucumber inbred lines were sown on the $1^{\text {st }}$ of January 2014 for the evaluation of resistance to powdery mildew and to produce all possible combination of first generation $\left(15 \mathrm{~F}_{1}\right)$ using half-diallel crossing (one direction) among the six parents. Planting was carried out under greenhouse conditions at Sabahia Horticultural Research Station, Alexandria Governorate, Egypt. After 42 days of sowing, plants with four leaves were inoculated by powdery mildew spores through artificial infection by inoculum spraying using infected leaves, as spore's source. These spores were used to make a suspension solution with a density of 20 spores/sight $(10 \times 10$ fold $)$ as described by Tang et al. (2003). Symptoms scale was given according to Zhang et al. (2011). Parents, used in the crossing program, were evaluated and classified according to their degree of resistance to powdery mildew disease, as illustrated by Mather and Jinks (1971).

In the following season, September 2014, seeds of the first generation $\left(15 \mathrm{~F}_{1}\right)$ and their parents $(6 \mathrm{P})$ were sown for self-pollination and crossed to produce the second generation $15\left(\mathrm{~F}_{2}\right), 15\left(\mathrm{BC}_{1}\right)$ and $15\left(\mathrm{BC}_{2}\right)$, in addition to new seeds of the $15 \mathrm{~F}_{1}$ 's and the 6 parents.

Seeds from each of the sixty six genotypes, $6(\mathrm{P}), 15$ $\left(\mathrm{F}_{1}\right), 15\left(\mathrm{~F}_{2}\right), 15\left(\mathrm{BC}_{1}\right)$ and $15\left(\mathrm{BC}_{2}\right)$, were evaluated under the greenhouse in Sabahia Horticulture Research Station, during the two seasons of February 2015 and September 2015. A Randomized Complete Blocks Design (RCBD), with three replicates, was used. Data recorded were fruits number /plant, total fruit yield/plant (g), average fruit weight $(\mathrm{g})$, fruit diameter $(\mathrm{cm})$ and fruit length $(\mathrm{cm})$.

Recorded data for the six populations; i.e., $\mathrm{P}_{1}, \mathrm{P}_{2}, \mathrm{~F}_{1}$, $\mathrm{F}_{2}, \mathrm{BC}_{1}$ and $\mathrm{BC}_{2}$ of each cross were statistically analyzed, using the combined analysis over two seasons, as outlined by Allard (1960). Types of gene action were calculated using the relationships given by Hayman (1958) and Gamble (1962).

\section{RESULTS AND DISCUSSION}

\section{Analyses of variances:}

Analyses of variances results revealed that there were highly significant differences among various evaluated genotypes for all studied characters (Table 1). These results indicated that the evaluated populations differed in their genetic potentials with respect to these studied traits.

Seasons mean squares were found to be highly significant for the studied characters fruit yield/plant, average fruit weight $(\mathrm{g})$ and fruit length $(\mathrm{cm})$. Such result indicated that there were some pronounced fluctuations in the environmental conditions from season to another throughout the two experiments of this investigation, affecting the general behavior of these characters. Variability between the two seasons might be related to the differences in temperature and other related climatic factors.

Interaction between genotypes and seasons $(\mathrm{G} \times \mathrm{S})$ reflected highly significant effects among all studied characters. Such a result, generally, suggested that the evaluated genotypes showed different responses when grown in different seasons and the genotype $\times$ environment interaction can have dramatic effects on most cucumber characters.

The obtained results, generally, agreed with these of Yadav et al. (2012) who reported that mean squares were highly significant for all studied traits, indicating the presence of a wide variability in cucumber genotypes. Feyzian et al. (2009) illustrated that the analysis of variance for all studied characters of melon indicated highly significant differences among genotypes.

Table 1. Combined analyses of variances (M.S. values) of yield and its components, and fruit characteristics of $6(\mathrm{P}), 15\left(\mathrm{~F}_{1}\right), 15\left(\mathrm{~F}_{2}\right), 15\left(\mathrm{BC}_{1}\right)$ and $15\left(\mathrm{BC}_{2}\right)$ of cucumber crosses over two growing seasons

\begin{tabular}{lcccccc}
\hline \multicolumn{1}{c}{ S.O.V } & D.F & $\begin{array}{c}\text { Total } \\
\text { yield/plant (g) }\end{array}$ & $\begin{array}{c}\text { Number of } \\
\text { fruits/ plant }\end{array}$ & $\begin{array}{c}\text { Average fruit } \\
\text { weight(g) }\end{array}$ & $\begin{array}{c}\text { Fruit } \\
\text { diameter } \\
\text { (cm) }\end{array}$ & $\begin{array}{c}\text { Fruit } \\
\text { length (cm) }\end{array}$ \\
\hline Blocks & 2 & $48732.98^{* *}$ & $14.15^{*}$ & 2.20 & $0.0952^{* *}$ & 0.461 \\
\hline Genotypes (G) & 65 & $143642.79^{* *}$ & $113.81^{* *}$ & $137.70^{* *}$ & $0.407 * *$ & $19.59 * *$ \\
\hline Seasons (S) & 1 & $120448.66^{* *}$ & 0.21 & $231.09 * *$ & 0.0159 & $4.59 * *$ \\
\hline G $\times$ S & 65 & $26940.25 * *$ & $7.61^{* *}$ & $28.35 * *$ & $0.065^{* *}$ & $2.20 * *$ \\
\hline Error & 262 & 7606.46 & 3.40 & 13.86 & 0.018 & 0.64 \\
\hline
\end{tabular}

*, ** Significant and highly significant at the 0.05 and 0.01 levels of probability, respectively. 
Mean performances of the various evaluated:

The mean values for the yield and its components are shown in Table (2). The genotypes $\mathrm{P}_{1} \times \mathrm{P}_{3}, \mathrm{P}_{2} \times \mathrm{P}_{4}$, $\mathrm{P}_{3} \times \mathrm{P}_{4}, \mathrm{P}_{4} \times \mathrm{P}_{5}, \mathrm{P}_{4} \times \mathrm{P}_{6}, \mathrm{BC}_{2}$ of $\left(\mathrm{P}_{4} \times \mathrm{P}_{6}\right)$ and $\mathrm{BC}_{2}$ of $\left(\mathrm{P}_{5} \times \mathrm{P}_{6}\right)$ gave the highest values for total yield/plant $(\mathrm{g})$ without significant difference between each other. The parental inbred line $\mathrm{P}_{1}$ gave the lowest total yield of all tested genotypes; and when it was as the recurrent parent, the total yield declined in most of the back crosses.
The mean values of number of fruits/plant ranged from 7.70 to 31.16 ; and the highest value of all tested genotypes appeared to be that recorded by the $\mathrm{F}_{1}$ hybrid $\mathrm{P}_{4} \times \mathrm{P}_{6}$, while the lowest number of fruits/plant was that recorded by the inbred line $\mathrm{P}_{1}$. The number of fruits/plant is one of the major components determining the total yield of the crop and the variation in this character might have been due to sex ratio and number of female flowers / node.

Table 2. Mean values of cucumber total yield/plant, number of fruits/plant, average fruit weight, fruit diameter and fruit length of the evaluated genotypes; the $6(P), 15\left(F_{1}\right), 15\left(F_{2}\right)$, $15\left(\mathrm{BC}_{1}\right)$ and $15\left(\mathrm{BC}_{2}\right)$, and a check cultivar over two growing seasons

\begin{tabular}{|c|c|c|c|c|c|}
\hline Genotype & $\begin{array}{c}\text { Total yield/plant } \\
\text { (g) }\end{array}$ & $\begin{array}{c}\text { Number of fruits/ } \\
\text { plant }\end{array}$ & $\begin{array}{c}\text { Average fruit } \\
\text { weight (g) }\end{array}$ & $\begin{array}{c}\text { Fruit diameter } \\
(\mathrm{cm})\end{array}$ & $\begin{array}{c}\text { Fruit length } \\
(\mathrm{cm})\end{array}$ \\
\hline \multicolumn{6}{|c|}{ Cross (1) $P_{1} \times P_{2}$} \\
\hline $\mathrm{P}_{1}$ & $431.01^{\mathrm{G}} *$ & $7.70^{\mathrm{D}}$ & $55.58^{\mathrm{abc}}$ & $3.08^{\mathrm{d}-\mathrm{j}}$ & $15.39^{\mathrm{d}-\mathrm{h}}$ \\
\hline $\mathrm{P}_{2}$ & $920.16^{\mathrm{f}-\mathrm{p}}$ & $16.87^{\mathrm{m}-\mathrm{w}}$ & $55.86^{\mathrm{ab}}$ & $2.93^{\mathrm{j}-\mathrm{p}}$ & $16.11^{\text {bcd }}$ \\
\hline $\mathrm{F}_{1}$ & $945.66^{\mathrm{e}-\mathrm{o}}$ & $20.16^{\mathrm{g}-1}$ & $47.44^{\mathrm{i}-\mathrm{o}}$ & $2.53^{z-D}$ & $17.31^{\mathrm{a}}$ \\
\hline $\mathrm{F}_{2}$ & $780.43^{\mathrm{r}-\mathrm{A}}$ & $14.75^{\mathrm{n}-\mathrm{x}}$ & $53.57^{\mathrm{a}-\mathrm{f}}$ & $2.65^{\mathrm{x}-\mathrm{A}}$ & $14.11^{\mathrm{i}-1}$ \\
\hline $\mathrm{F}_{1} \times \mathrm{P}_{1}\left(\mathrm{BC}_{1}\right)$ & $607.26^{\mathrm{EF}}$ & $12.58^{\mathrm{ABC}}$ & $49.57^{\mathrm{d}-\mathrm{k}}$ & $3.24^{\mathrm{a}-\mathrm{e}}$ & $15.78^{\mathrm{b}-\mathrm{e}}$ \\
\hline $\mathrm{F}_{1} \times \mathrm{P}_{2}\left(\mathrm{BC}_{2}\right)$ & $684.95^{\mathrm{Z}-\mathrm{F}}$ & $14.66^{\mathrm{u}-\mathrm{B}}$ & $47.76^{\mathrm{i}-\mathrm{o}}$ & $2.86^{\mathrm{n}-\mathrm{u}}$ & $15.4 \mathrm{~d}-\mathrm{h}$ \\
\hline \multicolumn{6}{|c|}{$\operatorname{Cross}(2) P_{1} \times P_{3}$} \\
\hline $\mathrm{P}_{1}$ & $431.01^{\mathrm{G}}$ & $7.70^{\mathrm{D}}$ & $55.58^{\mathrm{abc}}$ & $3.08^{\mathrm{d}-\mathrm{j}}$ & $15.39^{\mathrm{d}-\mathrm{h}}$ \\
\hline $\mathrm{P}_{3}$ & $780.55^{\mathrm{r}-\mathrm{A}}$ & $14.54^{\mathrm{v}-\mathrm{B}}$ & $53.51^{\mathrm{a}-\mathrm{f}}$ & $2.95^{\mathrm{j}-\mathrm{o}}$ & $13.13^{1-\mathrm{s}}$ \\
\hline $\mathrm{F}_{1}$ & $1163.18^{\mathrm{a}}$ & $26.16^{\mathrm{cd}}$ & $43.50^{\mathrm{m}-\mathrm{p}}$ & $2.95^{\mathrm{j}-0}$ & $15.96^{\mathrm{b}-\mathrm{e}}$ \\
\hline $\mathrm{F}_{2}$ & $824.75^{0-x}$ & $14.91^{\mathrm{t}-\mathrm{B}}$ & $50.84^{\mathrm{b}-\mathrm{k}}$ & $2.85^{\mathrm{n}-\mathrm{v}}$ & $14.38^{\mathrm{h}-\mathrm{k}}$ \\
\hline $\mathrm{F}_{1} \times \mathrm{P}_{1}\left(\mathrm{BC}_{1}\right)$ & $711.45^{\mathrm{x}-\mathrm{F}}$ & $11.87^{\mathrm{C}}$ & $47.81^{\mathrm{i}-\mathrm{o}}$ & $2.89^{1-\mathrm{s}}$ & $16.06^{\text {bcd }}$ \\
\hline $\mathrm{F}_{1} \times \mathrm{P}_{3}\left(\mathrm{BC}_{2}\right)$ & $691.70^{\mathrm{y}-\mathrm{F}}$ & $13.12^{\mathrm{z}-\mathrm{C}}$ & $46.21^{\mathrm{j}-\mathrm{p}}$ & $2.67^{\mathrm{w}-\mathrm{A}}$ & $14.57^{\mathrm{g}-\mathrm{k}}$ \\
\hline \multicolumn{6}{|c|}{ Cross (3) $\mathbf{P}_{1} \times \mathbf{P}_{4}$} \\
\hline $\mathrm{P}_{1}$ & $431.01^{\mathrm{G}}$ & $7.70^{\mathrm{D}}$ & $55.58^{\mathrm{abc}}$ & $3.08^{\mathrm{d}-\mathrm{j}}$ & $15.39^{\mathrm{d}-\mathrm{h}}$ \\
\hline $\mathrm{P}_{4}$ & $871.14^{\mathrm{k}-\mathrm{u}}$ & $18.16^{1-\mathrm{r}}$ & $48.49^{\mathrm{f}-\mathrm{m}}$ & $3.36^{\mathrm{a}}$ & $10.23^{\mathrm{F}}$ \\
\hline $\mathrm{F}_{1}$ & $914.26^{\mathrm{g}-\mathrm{p}}$ & $24.66^{\mathrm{de}}$ & $37.94^{\text {rst }}$ & $3.07^{\mathrm{d}-\mathrm{k}}$ & $15.65^{\mathrm{c}-\mathrm{f}}$ \\
\hline $\mathrm{F}_{2}$ & $739.91^{\mathrm{v}-\mathrm{D}}$ & $13.75^{\mathrm{y}-\mathrm{C}}$ & $54.19^{\mathrm{a}-\mathrm{e}}$ & $2.99^{\mathrm{h}-\mathrm{o}}$ & $13.26^{1-\mathrm{r}}$ \\
\hline $\mathrm{F}_{1} \times \mathrm{P}_{1}\left(\mathrm{BC}_{1}\right)$ & $625.04^{\mathrm{DEF}}$ & $12.58^{\mathrm{ABC}}$ & $45.63^{\mathrm{k}-\mathrm{p}}$ & $3.22^{\mathrm{a}-\mathrm{e}}$ & $15.00^{\mathrm{e}-\mathrm{i}}$ \\
\hline $\mathrm{F}_{1} \times \mathrm{P}_{4}\left(\mathrm{BC}_{2}\right)$ & $721.87^{\mathrm{x}-\mathrm{E}}$ & $17.00^{\mathrm{m}-\mathrm{w}}$ & $42.87^{\mathrm{opq}}$ & $3.06^{\mathrm{e}-1}$ & $10.73^{\mathrm{DEF}}$ \\
\hline \multicolumn{6}{|c|}{ Cross (4) $P_{1} \times P_{5}$} \\
\hline $\mathrm{P}_{1}$ & $431.01^{\mathrm{G}}$ & $7.70^{\mathrm{D}}$ & $55.58^{\mathrm{abc}}$ & $3.08^{\mathrm{d}-\mathrm{j}}$ & $15.39^{\mathrm{d}-\mathrm{h}}$ \\
\hline $\mathrm{P}_{5}$ & $757.29^{\mathrm{t}-\mathrm{B}}$ & $14.41^{\mathrm{w}-\mathrm{C}}$ & $52.64^{\mathrm{a}-\mathrm{i}}$ & $3.05^{\mathrm{e}-\mathrm{m}}$ & $10.68^{\mathrm{DEF}}$ \\
\hline $\mathrm{F}_{1}$ & $846.08^{\mathrm{m}-\mathrm{w}}$ & $16.95^{\mathrm{m}-\mathrm{w}}$ & $50.51^{\mathrm{c}-\mathrm{k}}$ & $2.53^{z-D}$ & $15.33^{\mathrm{d}-\mathrm{h}}$ \\
\hline $\mathrm{F}_{2}$ & $739.5^{\mathrm{v}-\mathrm{D}}$ & $14.08^{\mathrm{x}-\mathrm{C}}$ & $53.67^{\mathrm{a}-\mathrm{f}}$ & $2.53^{z-D}$ & $11.51^{\mathrm{x}-\mathrm{D}}$ \\
\hline $\mathrm{F}_{1} \times \mathrm{P}_{1}\left(\mathrm{BC}_{1}\right)$ & $595.62^{\mathrm{F}}$ & $12.41^{\mathrm{BC}}$ & $48.05^{\mathrm{h}-\mathrm{o}}$ & $3.18^{\mathrm{a}-\mathrm{g}}$ & $14.55^{\mathrm{g}-\mathrm{k}}$ \\
\hline $\mathrm{F}_{1} \times \mathrm{P}_{5}\left(\mathrm{BC}_{2}\right)$ & $850.37^{1-v}$ & $17.62^{\mathrm{m}-\mathrm{s}}$ & $49.06^{\mathrm{e}-\mathrm{k}}$ & $3.21^{\mathrm{a}-\mathrm{f}}$ & $10.78^{\mathrm{C}-\mathrm{F}}$ \\
\hline \multicolumn{6}{|c|}{$\operatorname{Cross}(5) P_{1} \times P_{6}$} \\
\hline $\mathrm{P}_{1}$ & $431.01^{\mathrm{G}}$ & $7.70^{\mathrm{D}}$ & $55.58^{\mathrm{abc}}$ & $3.08^{\mathrm{d}-\mathrm{j}}$ & $15.39^{\mathrm{d}-\mathrm{h}}$ \\
\hline $\mathrm{P}_{6}$ & $988.31^{\mathrm{c}-\mathrm{k}}$ & $17.37^{\mathrm{m}-\mathrm{t}}$ & $56.78^{\mathrm{a}}$ & $2.98^{\mathrm{j}-\mathrm{o}}$ & $13.58^{\mathrm{k}-\mathrm{p}}$ \\
\hline $\mathrm{F}_{1}$ & $1033.50^{b-g}$ & $23.12^{\mathrm{ef}}$ & $47.32^{\mathrm{i}-\mathrm{o}}$ & $3.26^{\mathrm{a}-\mathrm{d}}$ & $14.49^{g-k}$ \\
\hline $\mathrm{F}_{2}$ & $630.33^{\mathrm{C}-\mathrm{F}}$ & $13.20^{\mathrm{z}-\mathrm{C}}$ & $48.52^{\mathrm{f}-\mathrm{m}}$ & $2.83^{n-x}$ & $11.47^{\mathrm{x}-\mathrm{D}}$ \\
\hline $\mathrm{F}_{1} \times \mathrm{P}_{1}\left(\mathrm{BC}_{1}\right)$ & $625.87^{\mathrm{DEF}}$ & $12.75^{\mathrm{ABC}}$ & $49.55^{\mathrm{d}-\mathrm{k}}$ & $3.20^{\mathrm{a}-\mathrm{f}}$ & $15.76 b-e$ \\
\hline $\mathrm{F}_{1} \times \mathrm{P}_{6}\left(\mathrm{BC}_{2}\right)$ & $873.41^{\mathrm{k}-\mathrm{t}}$ & $16.41^{0-x}$ & $53.76^{\mathrm{a}-\mathrm{f}}$ & $2.76^{\mathrm{p}-\mathrm{y}}$ & $13.09^{1-\mathrm{s}}$ \\
\hline
\end{tabular}

*Means having an alphabetical letter (of the same order) in common within a particular column do not significantly differ from each other; using Duncan's Multiple Range Test, at 0.05 level. 
Table 2. continued

\begin{tabular}{|c|c|c|c|c|c|}
\hline Genotype & $\begin{array}{c}\text { Total } \\
\text { yield/plant (g) }\end{array}$ & $\begin{array}{c}\text { Number of } \\
\text { fruits/plant }\end{array}$ & $\begin{array}{l}\text { Average fruit } \\
\text { weight (g) }\end{array}$ & $\begin{array}{c}\text { Fruit } \\
\text { diameter }(\mathbf{c m})\end{array}$ & $\begin{array}{c}\text { Fruit } \\
\text { Length }(\mathrm{cm})\end{array}$ \\
\hline \multicolumn{6}{|c|}{ Cross (6) $P_{2} \times P_{3}$} \\
\hline $\mathrm{P}_{2}$ & $920.16^{\mathrm{f}-\mathrm{p}}$ & $16.87^{\mathrm{m}-\mathrm{w}}$ & $55.86^{\mathrm{ab}}$ & $2.93^{\mathrm{j}-\mathrm{p}}$ & $16.11^{\mathrm{bcd}}$ \\
\hline $\mathrm{P}_{3}$ & $780.55^{r-A}$ & $14.54^{\mathrm{v}-\mathrm{B}}$ & $53.51^{\mathrm{a}-\mathrm{f}}$ & $2.95^{j-0}$ & $13.13^{1-s}$ \\
\hline $\mathrm{F}_{1}$ & $950.97^{\mathrm{e}-\mathrm{n}}$ & $19.08^{\mathrm{h}-\mathrm{n}}$ & $50.15^{\mathrm{d}-\mathrm{k}}$ & $2.59^{\mathrm{y}-\mathrm{C}}$ & $15.77^{\mathrm{b}-\mathrm{e}}$ \\
\hline $\mathrm{F}_{2}$ & $653.29^{\mathrm{B}-\mathrm{F}}$ & $12.87^{\mathrm{ABC}}$ & $51.99^{\mathrm{a}-\mathrm{i}}$ & $2.54^{z-D}$ & $12.69^{0-w}$ \\
\hline $\mathrm{F}_{1} \times \mathrm{P}_{2}\left(\mathrm{BC}_{1}\right)$ & $743.62^{\mathrm{v}-\mathrm{D}}$ & $14.95^{\mathrm{t}-\mathrm{B}}$ & $49.87^{\mathrm{d}-\mathrm{k}}$ & $2.53^{z-D}$ & $12.98^{\mathrm{m}-\mathrm{t}}$ \\
\hline $\mathrm{F}_{1} \times \mathrm{P}_{3}\left(\mathrm{BC}_{2}\right)$ & $673.41^{\mathrm{A}-\mathrm{F}}$ & $15.12^{\mathrm{s}-\mathrm{A}}$ & $47.98^{i-0}$ & $2.63^{\mathrm{y}-\mathrm{B}}$ & $11.72^{\mathrm{v}-\mathrm{D}}$ \\
\hline \multicolumn{6}{|c|}{ Cross (7) $\mathbf{P}_{2} \times P_{4}$} \\
\hline $\mathrm{P}_{2}$ & $920.16^{\mathrm{f}-\mathrm{p}}$ & $16.87^{\mathrm{m}-\mathrm{w}}$ & $55.86^{\mathrm{ab}}$ & $2.93^{\mathrm{j}-\mathrm{p}}$ & $16.11^{\mathrm{bcd}}$ \\
\hline $\mathrm{P}_{4}$ & $871.14^{\mathrm{k}-\mathrm{u}}$ & $18.16^{1-\mathrm{r}}$ & $48.49^{\mathrm{f}-\mathrm{m}}$ & $3.36^{\mathrm{a}}$ & $10.23^{\mathrm{F}}$ \\
\hline $\mathrm{F}_{1}$ & $1095.67^{\mathrm{abc}}$ & $26.04^{\text {cd }}$ & $42.24^{\mathrm{pqr}}$ & $2.89^{\mathrm{k}-\mathrm{q}}$ & $16.75^{\mathrm{ab}}$ \\
\hline $\mathrm{F}_{2}$ & $880.41^{j-s}$ & $17.00^{\mathrm{m}-\mathrm{w}}$ & $51.51^{\mathrm{a}-\mathrm{j}}$ & $3.22^{\mathrm{a}-\mathrm{e}}$ & $12.34^{\mathrm{r}-\mathrm{z}}$ \\
\hline $\mathrm{F}_{1} \times \mathrm{P}_{2}\left(\mathrm{BC}_{1}\right)$ & $713.58^{x-F}$ & $14.75^{\mathrm{u}-\mathrm{B}}$ & $51.98^{\mathrm{a}-\mathrm{i}}$ & $2.49^{\mathrm{A}-\mathrm{D}}$ & $12.97^{\mathrm{m}-\mathrm{t}}$ \\
\hline $\mathrm{F}_{1} \times \mathrm{P}_{4}\left(\mathrm{BC}_{2}\right)$ & $884.08^{j-\mathrm{s}}$ & $20.75^{\mathrm{f}-\mathrm{k}}$ & $42.83^{\mathrm{opq}}$ & $3.19^{\mathrm{a}-\mathrm{f}}$ & $12.73^{\mathrm{o}-\mathrm{v}}$ \\
\hline \multicolumn{6}{|c|}{ Cross (8) $\mathbf{P}_{2} \times P_{5}$} \\
\hline $\mathrm{P}_{2}$ & $920.16^{\mathrm{f}-\mathrm{p}}$ & $16.87^{\mathrm{m}-\mathrm{w}}$ & $55.86^{\mathrm{ab}}$ & $2.93^{j-p}$ & $16.11^{\mathrm{bcd}}$ \\
\hline $\mathrm{P}_{5}$ & $757.29^{\mathrm{t}-\mathrm{B}}$ & $14.41^{\mathrm{w}-\mathrm{C}}$ & $52.64^{\mathrm{a}-\mathrm{i}}$ & $3.05^{\mathrm{e}-\mathrm{m}}$ & $10.68^{\mathrm{DEF}}$ \\
\hline $\mathrm{F}_{1}$ & $963.04^{\mathrm{d}-\mathrm{m}}$ & $18.16^{1-\mathrm{r}}$ & $53.41^{\mathrm{a}-\mathrm{f}}$ & $2.69^{t-z}$ & $13.94^{\mathrm{i}-\mathrm{m}}$ \\
\hline $\mathrm{F}_{2}$ & $764.33^{\mathrm{s}-\mathrm{B}}$ & $15.66^{\mathrm{r}-\mathrm{z}}$ & $47.54^{\mathrm{i}-\mathrm{o}}$ & $2.70^{\mathrm{s}-\mathrm{z}}$ & $12.05^{\mathrm{s}-\mathrm{B}}$ \\
\hline $\mathrm{F}_{1} \times \mathrm{P}_{2}\left(\mathrm{BC}_{1}\right)$ & $921.83^{\mathrm{f}-\mathrm{p}}$ & $18.70^{i-p}$ & $49.20^{\mathrm{d}-\mathrm{k}}$ & $2.68^{\mathrm{u}-\mathrm{z}}$ & $13.51^{\mathrm{k}-\mathrm{q}}$ \\
\hline $\mathrm{F}_{1} \times \mathrm{P}_{5}\left(\mathrm{BC}_{2}\right)$ & $766.70^{\mathrm{s}-\mathrm{B}}$ & $14.83^{t-B}$ & $52.28^{\mathrm{a}-\mathrm{i}}$ & $2.98^{\mathrm{i}-\mathrm{o}}$ & $11.16^{\mathrm{A}-\mathrm{F}}$ \\
\hline \multicolumn{6}{|c|}{ Cross (9) $P_{2} \times P_{6}$} \\
\hline $\mathrm{P}_{2}$ & $920.16^{f-p}$ & $16.87^{\mathrm{m}-\mathrm{w}}$ & $55.86^{\mathrm{ab}}$ & $2.93^{\mathrm{j}-\mathrm{p}}$ & $16.11^{\text {bcd }}$ \\
\hline $\mathrm{P}_{6}$ & $988.31^{\mathrm{c}-\mathrm{k}}$ & $17.37^{\mathrm{m}-\mathrm{t}}$ & $56.78^{\mathrm{a}}$ & $2.98^{\mathrm{j}-0}$ & $13.58^{\mathrm{k}-\mathrm{p}}$ \\
\hline $\mathrm{F}_{1}$ & $1038.58^{b-f}$ & $29^{\mathrm{b}}$ & $35.94^{\mathrm{t}}$ & $2.41^{\mathrm{D}}$ & $16.65^{\mathrm{abc}}$ \\
\hline $\mathrm{F}_{2}$ & $906.66^{\mathrm{h}-\mathrm{q}}$ & $16.25^{\mathrm{p}-\mathrm{y}}$ & $53.39^{\mathrm{a}-\mathrm{h}}$ & $3.10^{\mathrm{c}-\mathrm{j}}$ & $12.79^{\mathrm{n}-\mathrm{v}}$ \\
\hline $\mathrm{F}_{1} \times \mathrm{P}_{2}\left(\mathrm{BC}_{1}\right)$ & $832.37^{\text {n-x }}$ & $17.04^{\mathrm{m}-\mathrm{v}}$ & $49.29^{\mathrm{d}-\mathrm{k}}$ & $2.67^{\mathrm{v}-\mathrm{A}}$ & $13.85^{j-n}$ \\
\hline $\mathrm{F}_{1} \times \mathrm{P}_{6}\left(\mathrm{BC}_{2}\right)$ & $1024.91^{\mathrm{c}-\mathrm{h}}$ & $21.00^{f-j}$ & $49.29^{\mathrm{d}-\mathrm{k}}$ & $2.70^{r-z}$ & $11.33^{\mathrm{y}-\mathrm{E}}$ \\
\hline \multicolumn{6}{|c|}{ Cross (10) $\mathbf{P}_{3} \times \mathbf{P}_{4}$} \\
\hline $\mathrm{P}_{3}$ & $780.55^{\mathrm{r}-\mathrm{A}}$ & $14.54^{\mathrm{v}-\mathrm{B}}$ & $53.51^{\mathrm{a}-\mathrm{f}}$ & $2.95^{\mathrm{j}-\mathrm{o}}$ & $13.13^{1-s}$ \\
\hline $\mathrm{P}_{4}$ & $871.14^{\mathrm{k}-\mathrm{u}}$ & $18.16^{1-\mathrm{r}}$ & $48.49^{\mathrm{f}-\mathrm{m}}$ & $3.36^{\mathrm{a}}$ & $10.23^{\mathrm{F}}$ \\
\hline $\mathrm{F}_{1}$ & $1062.11^{\mathrm{a}-\mathrm{e}}$ & $21.20^{\mathrm{f}-\mathrm{i}}$ & $50.90^{\mathrm{b}-\mathrm{k}}$ & $2.89^{1-s}$ & $13.70^{j-0}$ \\
\hline $\mathrm{F}_{2}$ & $691.25^{\mathrm{y}-\mathrm{F}}$ & $12.75^{\mathrm{ABC}}$ & $54.5^{\mathrm{a}-\mathrm{d}}$ & $3.00^{\mathrm{g}-0}$ & $11.60^{\mathrm{w}-\mathrm{D}}$ \\
\hline $\mathrm{F}_{1} \times \mathrm{P}_{3}\left(\mathrm{BC}_{1}\right)$ & $749.62^{\mathrm{u}-\mathrm{C}}$ & $15.5^{\mathrm{s}-\mathrm{z}}$ & $49.01^{\mathrm{e}-\mathrm{k}}$ & $2.45^{\mathrm{BCD}}$ & $11.00^{\mathrm{B}-\mathrm{F}}$ \\
\hline $\mathrm{F}_{1} \times \mathrm{P}_{4}\left(\mathrm{BC}_{2}\right)$ & $898.41^{\mathrm{i}-\mathrm{r}}$ & $20.70^{\mathrm{g}-\mathrm{k}}$ & $43.71^{1-p}$ & $3.26^{\mathrm{a}-\mathrm{d}}$ & $11.9^{\mathrm{t}-\mathrm{B}}$ \\
\hline \multicolumn{6}{|c|}{ Cross (11) $P_{3} \times P_{5}$} \\
\hline $\mathrm{P}_{3}$ & $780.55^{\mathrm{r}-\mathrm{A}}$ & $14.54^{\mathrm{v}-\mathrm{B}}$ & $53.51^{\mathrm{a}-\mathrm{f}}$ & $2.95^{\mathrm{j}-\mathrm{o}}$ & $13.13^{1-\mathrm{s}}$ \\
\hline $\mathrm{P}_{5}$ & $757.29^{\mathrm{t}-\mathrm{B}}$ & $14.41^{\mathrm{w}-\mathrm{C}}$ & $52.64^{\mathrm{a}-\mathrm{i}}$ & $3.05^{\mathrm{e}-\mathrm{m}}$ & $10.68^{\mathrm{DEF}}$ \\
\hline$F_{1}$ & $826.03^{0-x}$ & $20.20^{\mathrm{g}-1}$ & $41.47^{\mathrm{p}-\mathrm{s}}$ & $2.73^{q-y}$ & $13.59^{k-p}$ \\
\hline $\mathrm{F}_{2}$ & $758.16^{t-B}$ & $14.08^{x-C}$ & $54.27^{\mathrm{a}-\mathrm{e}}$ & $2.43^{\mathrm{CD}}$ & $11.98^{\mathrm{t}-\mathrm{B}}$ \\
\hline $\mathrm{F}_{1} \times \mathrm{P}_{3}\left(\mathrm{BC}_{1}\right)$ & $734.08^{\mathrm{v}-\mathrm{D}}$ & $15.54^{\mathrm{s}-\mathrm{z}}$ & $47.37^{\mathrm{i}-0}$ & $2.55^{z-D}$ & $11.43^{x-D}$ \\
\hline $\mathrm{F}_{1} \times \mathrm{P}_{5}\left(\mathrm{BC}_{2}\right)$ & $890.12^{\mathrm{j}-\mathrm{r}}$ & $17.16^{\mathrm{m}-\mathrm{u}}$ & $52.51^{\mathrm{a}-\mathrm{i}}$ & $2.89^{\mathrm{k}-\mathrm{r}}$ & $13.48^{\mathrm{k}-\mathrm{q}}$ \\
\hline
\end{tabular}


Table 2. continued

\begin{tabular}{|c|c|c|c|c|c|}
\hline \multicolumn{6}{|c|}{$\operatorname{Cross}(12) P_{3} \times P_{6}$} \\
\hline $\mathrm{P}_{3}$ & $780.55^{\mathrm{r}-\mathrm{A}}$ & $14.54^{\mathrm{v}-\mathrm{B}}$ & $53.51^{\mathrm{a}-\mathrm{f}}$ & $2.95^{\mathrm{j}-\mathrm{o}}$ & $13.13^{1-\mathrm{s}}$ \\
\hline $\mathrm{P}_{6}$ & $988.31^{\mathrm{c}-\mathrm{k}}$ & $17.37^{\mathrm{m}-\mathrm{t}}$ & $56.78^{\mathrm{a}}$ & $2.98^{\mathrm{j}-\mathrm{o}}$ & $13.58^{\mathrm{k}-\mathrm{p}}$ \\
\hline $\mathrm{F}_{1}$ & $1001.63^{\mathrm{c}-j}$ & $27.16^{\mathrm{bc}}$ & $37.33^{\mathrm{st}}$ & $2.84^{\mathrm{n}-\mathrm{w}}$ & $13.86^{\mathrm{j}-\mathrm{n}}$ \\
\hline $\mathrm{F}_{2}$ & $777.91^{\mathrm{r}-\mathrm{A}}$ & $16.45^{0-x}$ & $47.50^{\mathrm{i}-\mathrm{o}}$ & $2.86^{\mathrm{n}-\mathrm{u}}$ & $12.42^{\mathrm{q}-\mathrm{y}}$ \\
\hline $\mathrm{F}_{1} \times \mathrm{P}_{3}\left(\mathrm{BC}_{1}\right)$ & $671.45^{\mathrm{A}-\mathrm{F}}$ & $13.16^{\mathrm{Z}-\mathrm{C}}$ & $50.95^{\mathrm{b}-\mathrm{k}}$ & $2.68^{\mathrm{u}-\mathrm{z}}$ & $11.72^{v-D}$ \\
\hline $\mathrm{F}_{1} \times \mathrm{P}_{6}\left(\mathrm{BC}_{2}\right)$ & $966.29^{\mathrm{d}-\mathrm{m}}$ & $19.37^{\mathrm{h}-\mathrm{m}}$ & $50.19^{\mathrm{d}-\mathrm{k}}$ & $3.21^{\mathrm{a}-\mathrm{e}}$ & $11.37^{\mathrm{y}-\mathrm{E}}$ \\
\hline \multicolumn{6}{|c|}{ Cross (13) $P_{4} \times P_{5}$} \\
\hline $\mathrm{P}_{4}$ & $871.14^{\mathrm{k}-\mathrm{u}}$ & $18.16^{1-\mathrm{r}}$ & $48.49^{\mathrm{f}-\mathrm{m}}$ & $3.36^{\mathrm{a}}$ & $10.23^{\mathrm{F}}$ \\
\hline $\mathrm{P}_{5}$ & $757.29^{\mathrm{t}-\mathrm{B}}$ & $14.41^{\mathrm{w}-\mathrm{C}}$ & $52.64^{\mathrm{a}-\mathrm{i}}$ & $3.05^{\mathrm{e}-\mathrm{m}}$ & $10.68^{\mathrm{DEF}}$ \\
\hline $\mathrm{F}_{1}$ & $1071.72^{\mathrm{a}-\mathrm{d}}$ & $22.41^{\mathrm{fg}}$ & $48.14^{g-n}$ & $2.83^{\mathrm{n}-\mathrm{x}}$ & $11.27^{\mathrm{z}-\mathrm{F}}$ \\
\hline $\mathrm{F}_{2}$ & $751.5^{\mathrm{t}-\mathrm{C}}$ & $15.54^{\mathrm{s}-\mathrm{z}}$ & $48.65^{\mathrm{f}-\mathrm{m}}$ & $2.74^{\mathrm{q}-\mathrm{y}}$ & $11.45^{\mathrm{x}-\mathrm{D}}$ \\
\hline $\mathrm{F}_{1} \times \mathrm{P}_{4}\left(\mathrm{BC}_{1}\right)$ & $804.08^{\mathrm{p}-\mathrm{z}}$ & $18.58^{\mathrm{j}-\mathrm{q}}$ & $43.21^{\text {nop }}$ & $3.16^{\mathrm{b}-\mathrm{i}}$ & $10.33^{\mathrm{EF}}$ \\
\hline $\mathrm{F}_{1} \times \mathrm{P}_{5}\left(\mathrm{BC}_{2}\right)$ & $902.20^{\mathrm{i}-\mathrm{q}}$ & $18.87^{\mathrm{i}-\mathrm{o}}$ & $49.79^{\mathrm{d}-\mathrm{k}}$ & $2.87^{\mathrm{n}-\mathrm{u}}$ & $12.49^{\mathrm{p}-\mathrm{x}}$ \\
\hline \multicolumn{6}{|c|}{ Cross (14) $P_{4} \times P_{6}$} \\
\hline $\mathrm{P}_{4}$ & $871.14^{\mathrm{k}-\mathrm{u}}$ & $18.16^{1-\mathrm{r}}$ & $48.49^{\mathrm{f}-\mathrm{m}}$ & $3.36^{\mathrm{a}}$ & $10.23^{\mathrm{F}}$ \\
\hline $\mathrm{P}_{6}$ & $988.31^{\mathrm{c}-\mathrm{k}}$ & $17.37^{\mathrm{m}-\mathrm{t}}$ & $56.78^{\mathrm{a}}$ & $2.98^{\mathrm{j}-\mathrm{o}}$ & $13.58^{\mathrm{k}-\mathrm{p}}$ \\
\hline $\mathrm{F}_{1}$ & $1145.04^{\mathrm{ab}}$ & $31.16^{\mathrm{a}}$ & $38.16^{\mathrm{qrst}}$ & $3.32^{\mathrm{ab}}$ & $13.21^{1-\mathrm{r}}$ \\
\hline $\mathrm{F}_{2}$ & $812.75^{\mathrm{p}-\mathrm{y}}$ & $16.08^{q-y}$ & $50.43^{\mathrm{c}-\mathrm{k}}$ & $3.17^{\mathrm{a}-\mathrm{h}}$ & $12.34^{\mathrm{r}-\mathrm{z}}$ \\
\hline $\mathrm{F}_{1} \times \mathrm{P}_{4}\left(\mathrm{BC}_{1}\right)$ & $790.54^{q-A}$ & $17.26^{\mathrm{m}-\mathrm{u}}$ & $45.94^{\mathrm{k}-\mathrm{p}}$ & $3.20^{\mathrm{a}-\mathrm{f}}$ & $10.61^{\mathrm{DEF}}$ \\
\hline $\mathrm{F}_{1} \times \mathrm{P}_{6}\left(\mathrm{BC}_{2}\right)$ & $1076.62^{\mathrm{a}-\mathrm{d}}$ & $20.62^{\mathrm{g}-1}$ & $52.62^{\mathrm{a}-\mathrm{i}}$ & $3.27^{\mathrm{abc}}$ & $11.85^{\mathrm{u}-\mathrm{C}}$ \\
\hline \multicolumn{6}{|c|}{ Cross (15) $P_{5} \times P_{6}$} \\
\hline $\mathrm{P}_{5}$ & $757.29^{t-B}$ & $14.41^{\mathrm{w}-\mathrm{C}}$ & $52.64^{\mathrm{a}-\mathrm{i}}$ & $3.05^{\mathrm{e}-\mathrm{m}}$ & $10.68^{\mathrm{DEF}}$ \\
\hline $\mathrm{P}_{6}$ & $988.31^{\mathrm{c}-\mathrm{k}}$ & $17.37^{\mathrm{m}-\mathrm{t}}$ & $56.78^{\mathrm{a}}$ & $2.98^{\mathrm{j}-\mathrm{o}}$ & $13.58^{\mathrm{k}-\mathrm{p}}$ \\
\hline $\mathrm{F}_{1}$ & $1017.83^{\mathrm{c}-\mathrm{i}}$ & $29^{\mathrm{b}}$ & $36.45^{\mathrm{t}}$ & $2.87^{\mathrm{m}-\mathrm{t}}$ & $14.68^{f-j}$ \\
\hline $\mathrm{F}_{2}$ & $674^{\mathrm{A}-\mathrm{F}}$ & $13.79^{\mathrm{y}-\mathrm{C}}$ & $48.84^{\mathrm{f}-1}$ & $3.02^{f-n}$ & $12.24^{\mathrm{r}-\mathrm{A}}$ \\
\hline $\mathrm{F}_{1} \times \mathrm{P}_{5}\left(\mathrm{BC}_{1}\right)$ & $723.37^{\mathrm{w}-\mathrm{E}}$ & $15.12^{\mathrm{s}-\mathrm{A}}$ & $48.03^{\mathrm{i}-\mathrm{o}}$ & $2.82^{0-x}$ & $12.77^{\mathrm{n}-\mathrm{v}}$ \\
\hline $\mathrm{F}_{1} \times \mathrm{P}_{6}\left(\mathrm{BC}_{2}\right)$ & $1141.25^{\mathrm{ab}}$ & $21.45^{\text {fgh }}$ & $53.46^{\mathrm{a}-\mathrm{g}}$ & $3.23^{\mathrm{a}-\mathrm{e}}$ & $12.84^{\mathrm{m}-\mathrm{u}}$ \\
\hline \multicolumn{6}{|c|}{ Check cultivar } \\
\hline Prince & $969^{\mathrm{d}-1}$ & $18.29^{\mathrm{k}-\mathrm{q}}$ & $53.70^{\mathrm{a}-\mathrm{f}}$ & $3.18^{\mathrm{a}-\mathrm{g}}$ & $10.73^{\mathrm{DEF}}$ \\
\hline
\end{tabular}

* Means having an alphabetical letter (of the same order) in common within a particular column do not significantly differ from each other; using Duncan's Multiple Range Test, at 0.05 level

Data in Table (2) illustrated that, the parental inbred lines $\mathrm{P}_{6}, \mathrm{P}_{2}$ and $\mathrm{P}_{1}$ had the highest fruit weight, with averages of $56.78,55.86$ and 55.58 , respectively. On the other hand, the lowest values were recorded by the $\mathrm{F}_{1}$ hybrid of the crosses $\mathrm{P}_{5} \times \mathrm{P}_{6}$ and $\mathrm{P}_{2} \times \mathrm{P}_{6} ; 36.45$ and 35.94 respectively.

Hossain et al. (2010) reported that the number of fruits/plant varied significantly among their tested accessions and ranged from 2.78 to 10.44 , and the cultivars showed significant differences in produced yield/plant, which ranged from $0.52 \mathrm{~kg}$ to $2.69 \mathrm{~kg}$.

Comparisons among means of the tested genotypes of fruit diameter and fruit length reflected significant differences in both traits (Table 2). Fruit diameter values of the various genotypes ranged from 2.41 to $3.36 \mathrm{~cm}$ and the highest value of all was recorded by the parental inbred line $\mathrm{P}_{4}(3.36 \mathrm{~cm})$; while, the lowest value was recorded by the $F_{1}$ hybrid of cross $\mathrm{P}_{2 \times} \mathrm{P}_{4}$ $(2.41 \mathrm{~cm})$. Concerning fruit length character, parental inbred line $\mathrm{P}_{2}$ had the longest fruit length among the parents $(16.11 \mathrm{~cm})$; while parental inbred line $\mathrm{P}_{4}$ had the shortest fruit length $(10.23 \mathrm{~cm})$. The $F_{1}$ hybrid of the cross $\mathrm{P}_{1} \times \mathrm{P}_{2}$ had the longest fruit of all evaluated genotypes $(17.32 \mathrm{~cm})$, followed by that of the cross $\mathrm{P}_{5} \times \mathrm{P}_{6}(14.68 \mathrm{~cm})$. The present results, generally, agreed with Khan et al. (2015) who reported that the detected variations in fruit diameter and fruit length of cucumber were great among all their studied genotypes.

\section{Resistance degrees to powdery mildew disease of the parental inbred lines and their $F_{1}$ hybrids:}

The results of determining the degrees of resistance of the six used parental inbred lines for powdery mildew disease under artificial infection, in (2014), are illustrated in Fig.(1): upon the detected reactions to artificial infection, the cucumber parental inbred lines were classified into four group i.e., $\mathrm{P}_{2}(99 \%)$ as high resistant (HR), $\mathrm{P} 1(90 \%)$ as resistant (R), $\mathrm{P}_{3}(65 \%)$ as 
tolerant $(\mathrm{T})$, and $\mathrm{P}_{4}(30 \%), \mathrm{P}_{5}(33 \%)$, and $\mathrm{P}_{6}(39 \%)$, as susceptible $(\mathrm{S})$.

According to the classification of resistance in Fig. (1), the parental inbred lines $P_{1}$ and $P_{2}$ were considered as (resistant parents), $\mathrm{P}_{3}$ (a tolerant parent) and $\mathrm{P}_{4}, \mathrm{P}_{5}$ and $\mathrm{P}_{6}$ as (susceptible parents), which were used in conducting half-diallel crosses program, aiming to produce resistant hybrids and to determine the types of gene action involved in the inheritance of powdery mildew resistance.

The results obtained on the reactions of powdery mildew resistance of the used 6 cucumber parental inbred lines and their $15 \mathrm{~F}_{1}$ hybrids, evaluated in 2015, are presented in Fig (2).

The obtained results showed that the $\mathrm{F}_{1}$ hybrid of the cross $\mathrm{P}_{2} \times \mathrm{P}_{4}$ was the only immune (I) $\mathrm{F}_{1}$ hybrid
$(100 \%)$; while $\mathrm{P}_{2}, \mathrm{P}_{1} \times \mathrm{P}_{2}, \mathrm{P}_{1} \times \mathrm{P}_{3}, \mathrm{P}_{2} \times \mathrm{P}_{5}$ genotypes were highly resistant (HR) for powdery mildew $98.33 \%$, $98.23 \%, 96.3$ and $95.83 \%$, respectively. The genotypes $\mathrm{P}_{1}, \quad \mathrm{P}_{1} \times \mathrm{P}_{6}, \quad \mathrm{P}_{2} \times \mathrm{P}_{3}$ and $\mathrm{P}_{3} \times \mathrm{P}_{5}$ showed relative weak infection degrees that ranged between $94-76 \%$, which might be considered as resistant $(\mathrm{R})$ genotypes; and, finally $\mathrm{P}_{3}, \mathrm{P}_{1} \times \mathrm{P}_{4}, \mathrm{P}_{1} \times \mathrm{P}_{5}, \mathrm{P}_{3} \times \mathrm{P}_{4}$ and $\mathrm{P}_{3} \times \mathrm{P}_{6}$ showed moderate degrees of resistance $75-51 \%$, making them as tolerant $(\mathrm{T})$ genotypes.

\section{Types of gene action:}

The estimated values of the various genetic parameters; i.e., population mean $(\mathrm{m})$, additive gene effect $(a)$, dominance gene effect $(d)$ and the three epistatic effects additive $\mathrm{x}$ additive $(a a)$, additive $\mathrm{x}$ dominance $(a d)$ and dominance $\mathrm{x}$ dominance $(d d)$ of the studied characters, are presented in Table (3).

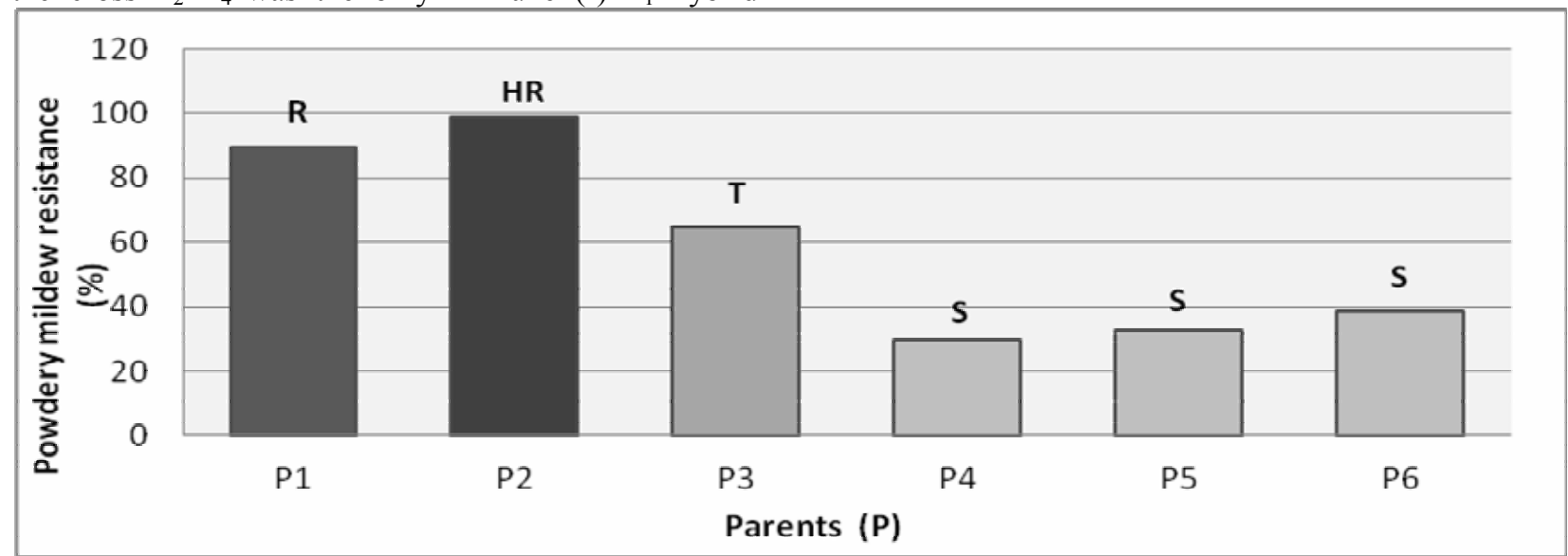

I= Immune (100\%), HR= Highly Resistant (99-95\%), R= Resistant (94-76\%), T= Tolerant (75-51\%), S= Susceptible (50-26\%).

Figure 1. Detected degrees of powdery mildew resistance of the 6 cucumber parental inbred lines under artificial infection conditions

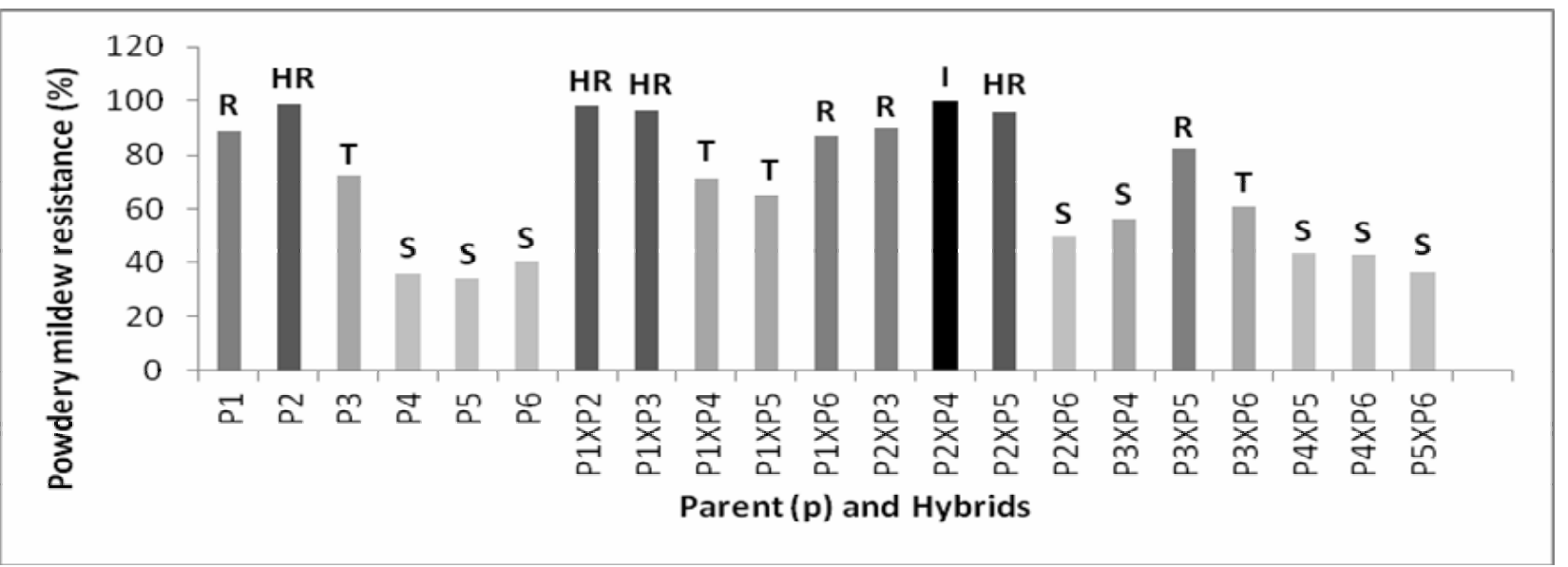

I= Immune (100\%), HR= Highly Resistant (99-95\%), R= Resistant (94-76\%), T= Tolerant (75-51\%), S= Susceptible (50-26\%).

Figure 2. Powdery mildew resistance evaluation of the cucumber 6 parents and their $15 F_{1}$ hybrids, under artificial infection conditions, in 2015 
Table 3. Estimated values of the six parameters of gene action involved on the inheritance of the studied characters of the possible 15 crosses among the 6 parental inbred lines of cucumber

\begin{tabular}{|c|c|c|c|c|c|c|}
\hline $\begin{array}{l}\text { Gene Action } \\
\text { parameter }\end{array}$ & $\begin{array}{c}\text { Total } \\
\text { yield/plant (g) }\end{array}$ & $\begin{array}{l}\text { Number of } \\
\text { fruits/ plant }\end{array}$ & $\begin{array}{c}\text { Average } \\
\text { fruit weight } \\
(\mathrm{g}) \\
\end{array}$ & $\begin{array}{c}\text { Fruit } \\
\text { diameter } \\
(\mathrm{cm})\end{array}$ & $\begin{array}{c}\text { Fruit } \\
\text { length }(\mathrm{cm})\end{array}$ & $\begin{array}{c}\text { Powdery } \\
\text { mildew } \\
\text { resistant \% }\end{array}$ \\
\hline \multicolumn{7}{|c|}{$\operatorname{Cross}(1) P_{1} \times P_{2}$} \\
\hline$m$ & $780.43 * *$ & $16.63 *$ & 53.58 & 2.65 & 14.12 & $84.33 * *$ \\
\hline$a$ & $-77.69 * *$ & -2.08 & 1.82 & 0.38 & 0.38 & $-4.67 * *$ \\
\hline$d$ & $-267.20 * *$ & $-4.13 * *$ & $-27.91 *$ & 1.14 & $7.46^{* *}$ & $28.62 * *$ \\
\hline$a a$ & -537.28 & $-12.00 * *$ & $-19.63 * *$ & 1.61 & $5.90 * *$ & $24.00 * *$ \\
\hline$a d$ & 166.88 & 2.50 & $1.96^{*}$ & 0.31 & 0.74 & $0.05 * *$ \\
\hline$d d$ & 1195.34 & $22.42 * *$ & $31.30 * *$ & -2.75 & $-2.13 * *$ & $-1.64 * *$ \\
\hline \multicolumn{7}{|c|}{ Cross (2) $P_{1} \times P_{3}$} \\
\hline$M$ & $824.75^{* *}$ & 14.92 & $50.84 * *$ & 2.86 & 14.38 & $78.33^{*}$ \\
\hline$a$ & $19.75 * *$ & -1.25 & 1.61 & 0.22 & 1.50 & $8.33 * *$ \\
\hline$d$ & $64.73 * *$ & 5.38 & $-26.36 * *$ & -0.38 & 5.44 & $43.08 * *$ \\
\hline$a a$ & $-492.67 * *$ & -9.67 & $-15.31 * *$ & -0.31 & 3.74 & $27.33 * *$ \\
\hline$a d$ & $194.52 * *$ & 2.17 & 0.57 & 0.16 & 0.37 & $0.00 * *$ \\
\hline$d d$ & $1224.26 * *$ & $34.25 * *$ & $23.36 * *$ & 1.14 & -4.55 & $-14.29 * *$ \\
\hline \multicolumn{7}{|c|}{ Cross (3) $P_{1} \times P_{4}$} \\
\hline$m$ & $739.92 * *$ & 13.75 & 54.20 & 3.00 & 13.27 & $72.00 * *$ \\
\hline$a$ & $-96.83 * *$ & -4.42 & 2.76 & 0.16 & 4.27 & $23.33 * *$ \\
\hline$d$ & $-2.65 * *$ & $15.90 *$ & $-53.86 * *$ & 0.44 & 1.24 & $34.46 * *$ \\
\hline$a a$ & $-265.83 * *$ & $4.17 *$ & $-39.77 * *$ & 0.58 & -1.60 & $25.33 * *$ \\
\hline$a d$ & $123.23 * *$ & 0.81 & -0.78 & 0.30 & 1.69 & $-3.15 * *$ \\
\hline$d d$ & $702.69 * *$ & $11.88 * *$ & $42.72 * *$ & -0.56 & 7.06 & $-70.79 * *$ \\
\hline \multicolumn{7}{|c|}{ Cross (4) $P_{1} \times P_{5}$} \\
\hline$m$ & $739.50 * *$ & 14.08 & 53.67 & 2.53 & 11.52 & 62.67 \\
\hline$a$ & $-254.75 * *$ & -5.21 & -1.00 & -0.02 & 13.77 & 12.67 \\
\hline$d$ & $185.93 * *$ & $9.65^{*}$ & $-24.04 * *$ & 2.13 & 6.90 & 50.83 \\
\hline$a a$ & $-66.00 * *$ & $3.75^{*}$ & $-20.44 * *$ & 2.66 & 4.60 & 48.00 \\
\hline$a d$ & $-91.61 * *$ & -1.85 & $-2.47 *$ & -0.03 & 1.41 & -14.06 \\
\hline$d d$ & $54.48 * *$ & $-7.79 * *$ & $35.48 * *$ & -4.25 & 1.47 & -92.33 \\
\hline \multicolumn{7}{|c|}{ Cross (5) $P_{1} \times P_{6}$} \\
\hline$m$ & $630.33 * *$ & 13.21 & 48.52 & 2.84 & 11.48 & $67.00 * *$ \\
\hline$a$ & $-247.54 * *$ & -3.67 & $-4.20 * *$ & 0.45 & 2.67 & $10.00 * *$ \\
\hline$d$ & $801.09 * *$ & $16.08 * *$ & $3.69 * *$ & 0.82 & 11.83 & $16.38 * *$ \\
\hline$a a$ & $477.25^{* *}$ & $5.50 * *$ & $12.55 * *$ & 0.59 & 11.82 & $-5.77 * *$ \\
\hline$a d$ & $31.11 * *$ & 1.17 & $-3.60 * *$ & 0.40 & 1.77 & $-14.21 * *$ \\
\hline$d d$ & $10.49 * *$ & $7.50 * *$ & $-12.17 * *$ & 0.06 & $-11.58 *$ & $46.58 * *$ \\
\hline \multicolumn{7}{|c|}{$\operatorname{Cross}(6) P_{2} \times P_{3}$} \\
\hline$m$ & $653.29 * *$ & 12.88 & $51.99 * *$ & 2.55 & 12.70 & 63.67 \\
\hline$a$ & $70.21 * *$ & -0.17 & $1.89 * *$ & -0.10 & 1.25 & 27.67 \\
\hline$d$ & $321.53 * *$ & 12.04 & $-16.76 * *$ & -0.22 & -0.23 & 18.69 \\
\hline$a a$ & $220.92 * *$ & 8.67 & $-12.23 * *$ & 0.13 & -1.37 & 14.00 \\
\hline$a d$ & $0.41 * *$ & -1.33 & $0.71 * *$ & -0.09 & -0.24 & 14.61 \\
\hline$d d$ & $547.66^{* *}$ & $0.75 * *$ & $26.20 * *$ & 0.64 & 12.75 & 67.82 \\
\hline
\end{tabular}


Table 3. continued

\begin{tabular}{|c|c|c|c|c|c|c|}
\hline \multicolumn{7}{|c|}{ Cross (7) $\mathbf{P}_{2} \times \mathbf{P}_{4}$} \\
\hline$m$ & $880.42 * *$ & 17.00 & $51.52 * *$ & 3.22 & 12.35 & $78.67 * *$ \\
\hline$a$ & $-170.50 * *$ & -6.00 & $9.15 * *$ & -0.70 & 0.24 & $43.00 * *$ \\
\hline$d$ & $-126.31 * *$ & $11.52 * *$ & $-26.37 * *$ & -1.74 & 5.60 & $-41.46^{* *}$ \\
\hline$a a$ & $-326.33 * *$ & $3.00 * *$ & $-16.44 * *$ & -1.49 & 2.03 & $-74.00 * *$ \\
\hline$a d$ & $-195.01 * *$ & -5.35 & $5.46 * *$ & -0.49 & -2.70 & $11.80 * *$ \\
\hline$d d$ & $1113.66^{* *}$ & $13.13 * *$ & $15.64 * *$ & 2.19 & $6.40^{*}$ & $166.92 * *$ \\
\hline \multicolumn{7}{|c|}{ Cross (8) $P_{2} \times P_{5}$} \\
\hline$m$ & $764.33 * *$ & 15.67 & $47.54 *$ & 2.70 & 12.05 & $73.33 * *$ \\
\hline$a$ & $155.13^{* *}$ & 3.88 & -3.08 & -0.30 & 2.35 & $29.56 * *$ \\
\hline$d$ & $444.07 * *$ & $6.94 * *$ & $12.22 * *$ & 0.23 & 1.68 & $-6.61 * *$ \\
\hline$a a$ & $319.75 * *$ & $4.42 * *$ & $12.78 * *$ & 0.54 & 1.13 & $-35.55 * *$ \\
\hline$a d$ & $73.69 * *$ & 2.65 & $-4.69 *$ & -0.24 & -0.37 & $-1.89 * *$ \\
\hline$d d$ & $-93.29 * *$ & $-3.88 * *$ & $0.15 * *$ & -0.50 & 4.21 & $103.22 * *$ \\
\hline \multicolumn{7}{|c|}{ Cross (9) $P_{2} \times P_{6}$} \\
\hline$m$ & $906.67 * *$ & 16.25 & $53.39 *$ & 3.10 & 12.79 & $62.00 * *$ \\
\hline$a$ & $-192.54 * *$ & -3.96 & $-0.005^{*}$ & -0.03 & 2.52 & $35.00 * *$ \\
\hline$d$ & $172.27 * *$ & $22.96 * *$ & $-36.77 * *$ & -2.19 & 1.02 & $15.93 * *$ \\
\hline$a a$ & $87.92 * *$ & $11.08 * *$ & $-16.38 * *$ & -1.64 & -0.78 & $35.33 * *$ \\
\hline$a d$ & $-158.47 * *$ & -3.71 & $0.45^{*}$ & -0.01 & 1.26 & $6.07 * *$ \\
\hline$d d$ & $183.14 * *$ & $5.08 * *$ & $3.74 * *$ & 1.62 & 13.40 & $-79.86 * *$ \\
\hline \multicolumn{7}{|c|}{ Cross (10) $P_{3} \times P_{4}$} \\
\hline$m$ & $691.25 * *$ & 12.75 & $54.50 * *$ & 3.01 & 11.61 & $48.33 * *$ \\
\hline$a$ & $-148.79 * *$ & -5.21 & $5.31 * *$ & -0.81 & -0.89 & $12.67 * *$ \\
\hline$d$ & $767.34 * *$ & $26.27^{*}$ & $-32.64 * *$ & -0.87 & 1.41 & $26.16 * *$ \\
\hline$a a$ & $531.08 * *$ & $21.42 *$ & $-32.54 * *$ & -0.60 & -0.61 & $24.00 * *$ \\
\hline$a d$ & $-103.50 * *$ & -3.40 & $2.80 * *$ & -0.61 & -2.35 & $-5.48 * *$ \\
\hline$d d$ & $-51.24 * *$ & $-18.71 * *$ & $50.89 * *$ & 1.27 & 5.58 & $-20.72 * *$ \\
\hline \multicolumn{7}{|c|}{$\operatorname{Cross}(11) P_{3} \times P_{5}$} \\
\hline$m$ & $758.17 * *$ & 14.08 & 54.28 & 2.44 & 11.98 & $55.33 * *$ \\
\hline$a$ & $-156.04 * *$ & -1.63 & -5.13 & -0.35 & -2.05 & $5.00 * *$ \\
\hline$d$ & $272.86 * *$ & $14.81 * *$ & $-28.94 * *$ & 0.87 & 3.58 & $38.30 * *$ \\
\hline$a a$ & $215.75^{* *}$ & $9.08 * *$ & $-17.33 * *$ & 1.14 & 1.89 & $10.00 * *$ \\
\hline$a d$ & $-167.67 * *$ & -1.69 & -5.57 & -0.29 & -3.28 & $-13.39 * *$ \\
\hline$d d$ & $-274.25 * *$ & $-5.12 * *$ & $6.65 * *$ & -0.54 & -0.72 & $30.60 * *$ \\
\hline \multicolumn{7}{|c|}{ Cross (12) $P_{3} \times P_{6}$} \\
\hline$m$ & $777.92 * *$ & 16.46 & 47.50 & 2.86 & 12.43 & $46.67 * *$ \\
\hline$a$ & $-294.83 * *$ & -6.21 & $0.77 * *$ & -0.53 & 0.35 & $30.33 * *$ \\
\hline$d$ & $281.04 * *$ & $10.46^{* *}$ & $-5.52 * *$ & 0.22 & -3.01 & $18.55 * *$ \\
\hline$a a$ & $163.83 * *$ & $-0.75 * *$ & $12.29 * *$ & 0.34 & -3.51 & $14.00 * *$ \\
\hline$a d$ & $-190.96 * *$ & -4.79 & $2.40 * *$ & -0.52 & 0.57 & $14.46^{* *}$ \\
\hline$d d$ & $332.81 * *$ & $21.92 * *$ & $-29.63 * *$ & -0.52 & 11.77 & $19.83 * *$ \\
\hline \multicolumn{7}{|c|}{ Cross (13) $P_{4} \times P_{5}$} \\
\hline$m$ & $751.50 * *$ & 15.54 & $48.66^{* *}$ & 2.75 & 11.45 & $34.00 * *$ \\
\hline$a$ & $-98.13 * *$ & $-0.29 *$ & $-6.57 *$ & 0.30 & -2.16 & $8.00 * *$ \\
\hline$d$ & $664.09 * *$ & $18.88 * *$ & $-11.03 * *$ & 0.62 & $0.66^{* *}$ & $-6.62 * *$ \\
\hline$a a$ & $406.58 * *$ & $12.75 * *$ & $-8.60 * *$ & 1.09 & $-0.16^{* *}$ & $-14.67 * *$ \\
\hline$a d$ & $-155.05 * *$ & $-2.17 *$ & $-4.50 *$ & 0.15 & -1.94 & $7.76^{* *}$ \\
\hline$d d$ & $-47.28 * *$ & $-10.25 * *$ & $20.00 * *$ & -1.26 & $-2.02 * *$ & $52.17 * *$ \\
\hline
\end{tabular}


Table 3. continued

\begin{tabular}{ccccccc}
\hline \multicolumn{7}{c}{ Cross (14) $\mathbf{P}_{\mathbf{4}} \times \mathbf{P}_{\mathbf{6}}$} \\
\hline$m$ & $812.75^{* *}$ & $16.08^{* *}$ & 50.44 & 3.18 & 12.34 & $28.00^{* *}$ \\
\hline$a$ & $-286.08^{* *}$ & -3.36 & -6.68 & -0.07 & -1.24 & $2.67^{* *}$ \\
\hline$d$ & $698.64^{* *}$ & $24.84^{* *}$ & $-19.09^{* *}$ & 0.41 & -3.12 & $-28.50^{* *}$ \\
\hline$a a$ & $483.33^{* *}$ & $11.45^{* *}$ & $-4.62^{* *}$ & 0.25 & -4.43 & $-33.33^{* *}$ \\
\hline$a d$ & $-227.50^{* *}$ & -3.76 & -2.54 & -0.26 & 0.44 & $4.94^{* *}$ \\
\hline$d d$ & $-68.12^{* *}$ & $10.66^{* *}$ & $-10.92^{* *}$ & -0.22 & $9.74^{*}$ & $117.13^{* *}$ \\
\hline \multicolumn{7}{c}{$\mathbf{C r o s s} \mathbf{( 1 5 )} \mathbf{P}_{5} \times \mathbf{P}_{\mathbf{6}}$} \\
\hline$m$ & $-13.79^{*}$ & 48.84 & 3.03 & 12.25 & $39.33^{* *}$ \\
\hline$a$ & $-417.88^{* *}$ & -6.33 & -5.43 & -0.42 & -0.07 & $4.67^{* *}$ \\
\hline$d$ & $1178.28^{* *}$ & $31.10^{* *}$ & -10.62 & -0.13 & 4.80 & $-39.96^{* *}$ \\
\hline$a a$ & $1033.25^{* *}$ & $18.00^{* *}$ & 7.65 & 0.01 & 2.25 & $-38.67^{* *}$ \\
\hline$a d$ & $-302.37^{* *}$ & -4.85 & -3.36 & -0.46 & 1.39 & $7.18^{* *}$ \\
\hline$d d$ & $-981.23^{* *}$ & $-1.38^{* *}$ & -28.33 & -0.34 & $0.14^{*}$ & $69.26^{* *}$ \\
\hline
\end{tabular}

*,** Significant and highly significant at the 0.05 and 0.01 level of probability, respectively. ( $m, a, d, a a, a d$ and $d d=$ population mean, additive, dominant, additive $\mathrm{x}$ additive, additive $\mathrm{x}$ dominant and dominant $\mathrm{x}$ dominant gene action, respectively).

The estimated values of mean effects $(m)$ on total yield/plant (g), clearly, appeared to be significant in all crosses, indicating that population mean might be considered as an effective selection indicator for this trait. The additive gene actions $(a)$ exhibited highly significant, negative or positive, values in the various tested crosses, whereas the dominance effects $(d)$ values were found to be highly significant positive in most of the crosses and highly significant but negative in the crosses $\mathrm{P}_{1} \times \mathrm{P}_{2}, \mathrm{P}_{1} \times \mathrm{P}_{4}$ and $\mathrm{P}_{2} \times \mathrm{P}_{4}$. The additive $\mathrm{x}$ additive interaction $(a a)$ was found to be positively high significant in most of the tested crosses, but not in the five crosses $\mathrm{P}_{1} \times \mathrm{P}_{2}, \mathrm{P}_{1} \times \mathrm{P}_{3}, \mathrm{P}_{1} \times \mathrm{P}_{4}, \mathrm{P}_{1} \times \mathrm{P}_{5}$ and $\mathrm{P}_{2} \times \mathrm{P}_{4}$ which suggested the importance of additive $\mathrm{x}$ additive epistatic type of gene action on the inheritance of total yield. The additive $\mathrm{x}$ dominance type of interaction $(a d)$ was also found to be positively high significant for $\mathrm{P}_{1} \times \mathrm{P}_{2}, \mathrm{P}_{1} \times \mathrm{P}_{3}$, $\mathrm{P}_{1} \times \mathrm{P}_{4}, \mathrm{P}_{1} \times \mathrm{P}_{6}, \mathrm{P}_{2} \times \mathrm{P}_{3}$ and $\mathrm{P}_{2} \times \mathrm{P}_{5}$; while, it was negatively high significant for the rest of the tested crosses. This result suggested that the selection for this trait in the early generations would be not effective because of the high significant effect of the additive $\mathrm{x}$ dominance interaction $(a d)$. The dominance $\mathrm{x}$ dominance interaction ( $d d$ ) was found to be negatively high significant in the crossed $\mathrm{P}_{2} \times \mathrm{P}_{5}, \mathrm{P}_{3} \times \mathrm{P}_{4}, \mathrm{P}_{3} \times \mathrm{P}_{5}, \mathrm{P}_{4} \times \mathrm{P}_{5}$, $\mathrm{P}_{4} \times \mathrm{P}_{6}$ and $\mathrm{P}_{5} \times \mathrm{P}_{6}$, and positively significant in the rest. This result indicated also that the dominance gene action $(d)$ was important on the inheritance of this trait.

Concerning number of fruits/plant, data in Table (3) illustrated that the estimated values of mean effects were found to be insignificant in all crosses; except $\mathrm{P}_{1} \times \mathrm{P}_{2}, \mathrm{P}_{4} \times \mathrm{P}_{6}$ and $\mathrm{P}_{5} \times \mathrm{P}_{6}$ crosses; indicating that the population mean may not be as effective as an indicator for selection for this trait. The additive gene action $(a)$ exhibited a significant value in only one cross $\left(\mathrm{P}_{4} \times \mathrm{P}_{5}\right)$.
On the other hand, the dominance gene effect $(d)$ exhibited highly significant values in most of the tested crosses, indicating that the dominance gene effect had the main role on the inheritance of this character. The additive $\mathrm{x}$ additive interaction $(a a)$ was found to be positively high significant in most crosses; except $\mathrm{P}_{1} \times \mathrm{P}_{2}$ and $\mathrm{P}_{3} \times \mathrm{P}_{6}$ crosses, which recorded negative significance values. Insignificant values were obtained in the two crosses $\mathrm{P}_{1} \times \mathrm{P}_{3}$ and $\mathrm{P}_{2} \times \mathrm{P}_{3}$. The additive $\mathrm{x}$ dominance epistatic type of gene action (ad) was found to be insignificant in all crosses, except in the cross $\mathrm{P}_{4} \times \mathrm{P}_{5}$; while, the dominance $\mathrm{x}$ dominance interaction $(d d)$ was found to be negatively high significant in the crosses $\mathrm{P}_{1} \times \mathrm{P}_{5}, \quad \mathrm{P}_{2} \times \mathrm{P}_{5}, \quad \mathrm{P}_{3} \times \mathrm{P}_{4}, \quad \mathrm{P}_{3} \times \mathrm{P}_{5}, \quad \mathrm{P}_{4} \times \mathrm{P}_{5}$ and $\mathrm{P}_{5} \times \mathrm{P}_{6}$ and positively significant in the rest of the tested crosses. These results indicated that the dominance $\mathrm{x}$ dominance interaction $(d d)$ seemed to be more important on the inheritance of number of fruits/plant character.

Obtained results for average fruit weight $(\mathrm{g})$ declared that the estimate values for mean effect were found to be significant in the crosses $\mathrm{P}_{1 \times} \mathrm{P}_{3}, \mathrm{P}_{2 \times} \mathrm{P}_{3}, \mathrm{P}_{2 \times} \times \mathrm{P}_{4}$, $\mathrm{P}_{2 \times} \mathrm{P}_{5}, \mathrm{P}_{2 \times} \mathrm{P}_{6}, \mathrm{P}_{3 \times} \mathrm{P}_{4}$ and $\mathrm{P}_{4 \times} \mathrm{P}_{5}$. The additive gene action (a) was found to be positively high significant in $\mathrm{P}_{2 \times} \mathrm{P}_{3}$, $\mathrm{P}_{2 \times} \mathrm{P}_{4}, \mathrm{P}_{3 \times} \mathrm{P}_{4}$ and $\mathrm{P}_{3 \times} \times \mathrm{P}_{6}$ crosses; while, the dominance effects $(d)$ exhibited negatively high significant values in most of the tested crosses; except the two crosses $\mathrm{P}_{1} \times \mathrm{P}_{6}$ and $\mathrm{P}_{2} \times \mathrm{P}_{5}$, which have positively significant value. The additive $\mathrm{x}$ additive interaction $(a a)$ was found to be negatively high significant in most of the tested crosses; only the three crosses $\mathrm{P}_{1} \times \mathrm{P}_{6}, \mathrm{P}_{2} \times \mathrm{P}_{5}$ and $\mathrm{P}_{3} \times \mathrm{P}_{6}$ appeared to have positively significant values. The additive $\mathrm{x}$ dominance interaction $(a d)$ was found to be positively significant in $\mathrm{P}_{1} \times \mathrm{P}_{2}, \mathrm{P}_{2} \times \mathrm{P}_{3} \mathrm{P}_{2} \times \mathrm{P}_{4}, \mathrm{P}_{2} \times \mathrm{P}_{6}$ $\mathrm{P}_{3} \times \mathrm{P}_{4}$, and $\mathrm{P}_{3} \times \mathrm{P}_{6}$ crosses. The dominance $\mathrm{x}$ dominance interaction $(d d)$ was estimated by positively high significant values in all crosses, with the exception of 
the crosses $\mathrm{P}_{1 \times} \mathrm{P}_{6}, \quad \mathrm{P}_{3 \times} \mathrm{P}_{6}, \mathrm{P}_{4 \times} \mathrm{P}_{6}$ and $\mathrm{P}_{5 \times} \mathrm{P}_{6}$; which indicated that dominance $\mathrm{x}$ dominance interaction $(d d)$ appeared to be more important on the inheritance of this character.

With reference to fruit diameter trait, all estimated values for the various types of gene action; i.e., mean effects $(m)$, additive $(a)$, dominance $(d)$, additive $\mathrm{x}$ additive $(a a)$, additive $\mathrm{x}$ dominance $(a d)$ and dominance $\mathrm{x}$ dominance $(d d)$ showed insignificant values in all crosses; which reflected the absence of a distinguished role for the involved parameter on the inheritance of this character.

For fruit length, the additive $(a)$ and dominance $(d)$ gene action appeared to have insignificant estimates in all tested crosses. The additive $\mathrm{x}$ additive interaction ( $a$ a ) was also found to be insignificant in all studied crosses, except the cross $\mathrm{P}_{1} \times \mathrm{P}_{2}$. Also, the additive $\mathrm{x}$ dominance interaction (ad) exhibited insignificant values in all crosses. The dominance $\mathrm{x}$ dominance interaction $(d d)$ values were found to be positively high significant in $\mathrm{P}_{2} \times \mathrm{P}_{4}, \mathrm{P}_{4} \times \mathrm{P}_{6}$ and $\mathrm{P}_{5} \times \mathrm{P}_{6}$, and negatively significant in $\mathrm{P}_{1} \times \mathrm{P}_{6}, \mathrm{P}_{1} \times \mathrm{P}_{2}$ and $\mathrm{P}_{4} \times \mathrm{P}_{5}$ crosses; indicating that $(d d)$ was more important on the inheritance of this character than $(a a)$ and $(a d)$.

The estimated values of the different types of gene action on the inheritance of powdery mildew resistance are presented in Table (3). Values of mean effects were found to be highly significant in all crosses; except two crosses $\mathrm{P}_{1} \times \mathrm{P}_{5}$ and $\mathrm{P}_{2} \times \mathrm{P}_{3}$. Also, additive gene action $(a)$ exhibited positively significant values in most crosses, which indicated that additive effects played a main role on the inheritance of this trait. The dominance gene effect were found to be positively high significant in most crosses; except $\mathrm{P}_{2} \times \mathrm{P}_{4}, \mathrm{P}_{2} \times \mathrm{P}_{5}, \mathrm{P}_{4} \times \mathrm{P}_{5}, \mathrm{P}_{4} \times \mathrm{P}_{6}$ and $\mathrm{P}_{5} \times \mathrm{P}_{6}$, which appeared negatively significant. The additive $\mathrm{x}$ additive interaction $(a a)$ was found to be positively high significant in most crosses, except $\mathrm{P}_{1} \times \mathrm{P}_{6}, \quad \mathrm{P}_{2} \times \mathrm{P}_{4}, \quad \mathrm{P}_{2} \times \mathrm{P}_{5}, \quad \mathrm{P}_{4} \times \mathrm{P}_{5}, \quad \mathrm{P}_{4} \times \mathrm{P}_{6}$ and $\mathrm{P}_{5} \times \mathrm{P}_{6}$. The additive $\mathrm{X}$ dominance interaction $(\mathrm{ad})$ appeared to be positively high significant in most crosses, except $\mathrm{P}_{1} \times \mathrm{P}_{4}, \mathrm{P}_{1} \times \mathrm{P}_{6}, \mathrm{P}_{2} \times \mathrm{P}_{5}, \mathrm{P}_{3} \times \mathrm{P}_{4}$ and $\mathrm{P}_{3} \times \mathrm{P}_{5}$ while, it was insignificant in $\mathrm{P}_{1} \times \mathrm{P}_{5}$ and $\mathrm{P}_{2} \times \mathrm{P}_{3}$. The dominance $\mathrm{x}$ dominance interaction $(d d)$ was estimated by positively high significant values in most crosses; only five crosses gave negative significance values.

Results of the present study seemed to have some agreement with those reported by Sarkar and Sirohi (2011), who indicated that dominance effects on fruit weight, fruit diameter, fruit length, number of fruits/plant and fruit yield/plant were higher than additive effects in cucumber. Also, Abou Kamer (2014), explained that the dominant $\mathrm{x}$ dominant gene action; played an important role on the inheritance of the traits average number of fruits/plant and total fruit yield/plant of melon. Shahi et al. (2005) also, mentioned that additive $\mathrm{x}$ additive type of epistasis was found significant on fruit diameter and number of fruits. The additive $\mathrm{x}$ dominance and dominance $\mathrm{x}$ dominance types of epistasis were significant on all the characters, except fruit weight. The additive and dominance components of genetic variance appeared to be highly significant on all studied characters; except average fruit weight, which showed insignificant additive effects.

It could be concluded that the two hybrids $\mathrm{P}_{1} \times \mathrm{P}_{3}$ and $\mathrm{P}_{2} \times \mathrm{P}_{4}$ are promising for resistance to powdery mildew disease, in addition, they have given the best results for all the studied characters during both seasons.

\section{REFERENCES}

Abd El-Sayed, M.H. 2002. Studies on powdery mildew disease of cucurbits under protected cultivation. M.Sc. Thesis, Fac. Agric., Cairo. Univ., 180 pp.

Abou Kamer, M.E. 2011. Studies on heterosis and nature of gene action and their effects on yield and fruit quality in sweet melon (Cucumis Melo, L). M.Sc. Thesis, Fac. Agric. (Saba Basha) Alexandria University, Egypt.

Abou Kamer, M.E. 2014. Inheritance of some important characters to improve sweet melon (Cucumis melo, L.) fruits. Ph. D. Thesis, Fac. Agric. (Saba Basha) Alexandria University, Egypt.

Allard, R.W. 1960. Principles of plant breeding .London, John Wiley and Sons, pp.458.

El-Mgihawry, A., M.M.M. Abel-Salam, S.M.H. Sarg, Y.T.E. El-Lithy and M.A.F. El-Tahawy 2008. Types of gene action, combining ability and reciprocal differences for some important characters in summer squash (Cucurbita pepoL.) Egypt. J. plant Breed. 12(2):133-142.

Feyzian, E., H. Dehghani, A.M. Rezai and M. Jalali-Javaran 2009. Diallel cross analysis for maturity and yield related traits in melon (Cucumis melo L.). Euphytica 168: 215223.

Gamble, E.E. 1962. Gene effects in corn (Zea mays L.) I. Separation and relative importance of gene effects for yield. Canad. J. plant Sci. 42:339-348.

Hayman, B.I. 1958. The separation of epistasic from additive and dominance variation in generation means. Heredity 12:371-390.

Hossain, M.F., M.G. Rabbani, M.A. Hakim, A.S.M. Amanullah and A.S.M. Ahsanullah 2010 Study on varitability character association and yield performance of cucumber (Cucumis sativusL.) Bangladesh research publications journal 4 (3), Page: 297-311.

Khan Z., A.H. Shah, R. Gul, A. Majid, U. Khan, and H. Ahmad 2015. Morpho-agronomic characterization of cucumber germplasm for yield and yield associated traits. International Journal of Agronomy and Agricultural Research (IJAAR) ISSN: 2223-7054 6(1):1-6. 
Mather, K. and Y.L. Jinks 1971. Biometrical genetics, the study of continuous variation. Cornell State.Univ . Press, USA.

Sarkar, M. and P.S. Sirohi 2011. Diallel analysis of quantitative characters in cucumber (Cucumis sativusL.) Vegetable Science 38(1): 73-75.

Shahi, B.P., J. Dixit and P.K. Singh 2005. Additive, dominance and epistatic variation for fruit yield and its component traits in cucumber Veg. Sci. 32(1): 27-29.
Tang, R.X.Z., T. Hu and K. Cao 2003. Control effect of the extracts from Rheum palmatum on powdery mildew of cucumber. J. Anhui. Agric. Univ. 30(4): 363 - 366.

Yadav, Y.C., S. Kumar and R. Singh 2012. studies on genetic variability, heritability and genetic advance in cucumber (Cucumis sativus L.) HortFlora Research Spectrum, 1(1): 34-37.

Zhang, S., G.E. Vallad, T.L. White and C.H. Huang 2011. Evaluation of microbial products for management of powdery mildew on summer squash and cantaloupe in Florida. Plant Disease, Vol. 95, No. 4: 461- 467.

\title{
الملضص العرب - (المي \\ توارث صفة المقاومة لمرض البيلض الهيقي وصضن المفلت الفتصالية في الخيار
}

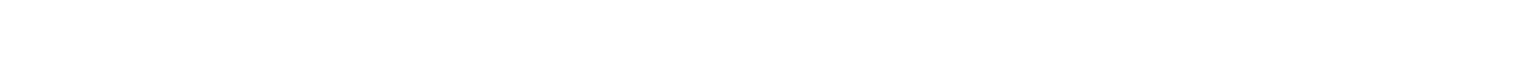

\author{
سورن مسعد العبد
}

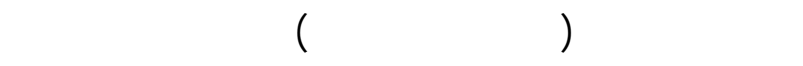

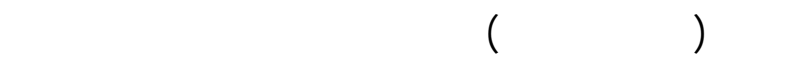

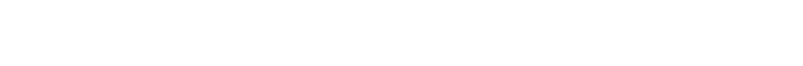

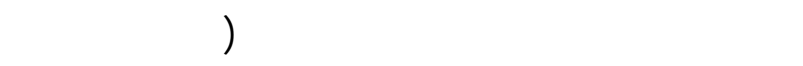

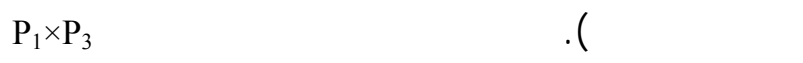
كان $\mathrm{P}_{2} \times \mathrm{P}_{4}$,

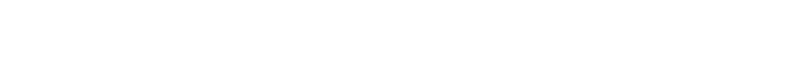

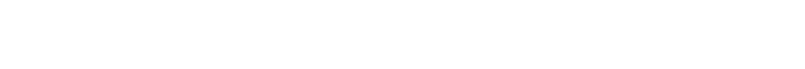

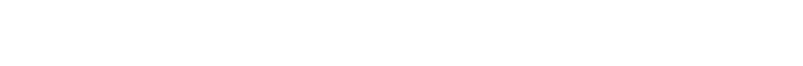

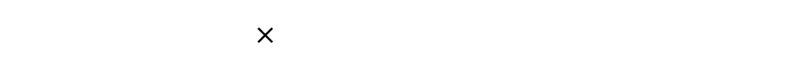

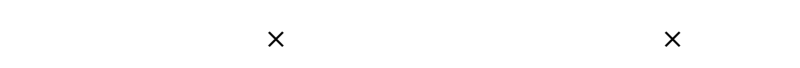

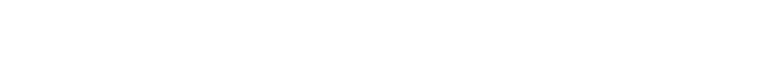
المبكرة من البرنلمج الإنتخابي.
لجريت هذه الدرلسة ف ي مطط عة بح ـوث الب ساتين

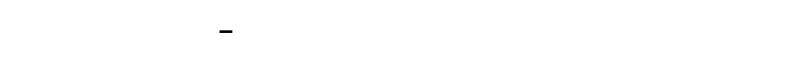
درلمة الفعل الجيني لبعض الصفلت الاقتصاية وكذك صفة الحفة

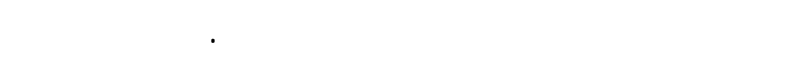

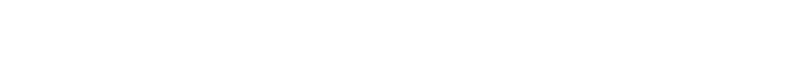

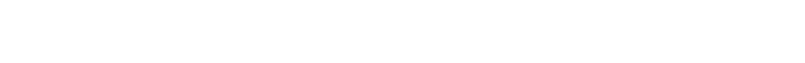
ودون التهجينت العكسية. ولقد قم لجراء القيم المرض في

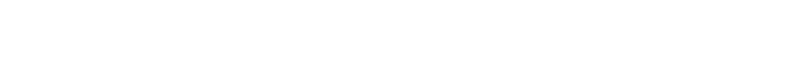
شهر يناير لعلم عا.ب حيث قم الحصول علي بذور الجيل

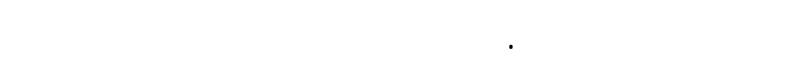
لإجراء التلقيح الذاتي والرجعي لكلا الابوين في لكل تهجين الإنين

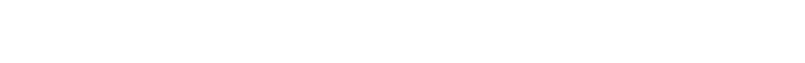

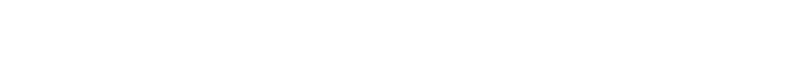

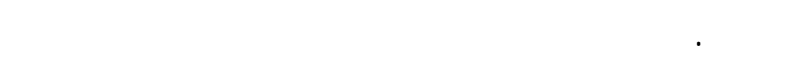

